\title{
Variação de IMC, padrões alimentares e atividade física entre adultos de 21 a 44 anos
}

\author{
BMI variation, dietary patterns and physical activity \\ among adults aged 21-44 years
}

Iolanda Karla Santana dos Santos (https://orcid.org/0000-0003-3347-8396) 1,2

Wolney Lisbôa Conde (https://orcid.org/0000-0003-0493-134X) ${ }^{1}$

${ }^{1}$ Faculdade de Saúde Pública, Universidade de São Paulo. Av. Dr. Arnaldo 715, Cerqueira César. 01246904 São Paulo SP Brasil. iolanda.santos@usp.br

${ }^{2}$ Fundação Universidade

Federal do ABC. Santo

André SP Brasil.

\begin{abstract}
The scope of this article is to describe the association between BMI variation, eating patterns and physical activity among adults between 21 and 44 years of age from 2007 to 2012. It is a cross-sectional study using the VIGITEL database. Eating patterns were identified with Principal Components Analysis for the period from 2007 to 2012. Components with eigenvalues $>1.0$ were retained and factor loadings greater than $|0.3|$ were highlighted. For each individual, a score was calculated per pattern. The Body Mass Index ( $\triangle B M I)$ difference variable was then created. Linear regression with $\triangle B M I$ outcome and Poisson regression with obesity outcomes were conducted. Four eating patterns were retained: Prudent, Transition, Western and Traditional. After multivariate adjustment, $\triangle B M I$ was inversely associated with the habit of leisure-time physical activity. Obesity revealed a positive association with the Western pattern, watching television $\geq 3$ hours a day and physical inactivity. Obesity was inversely associated with the Prudent pattern, the Traditional pattern, the practice of leisure-time physical activity and the habit of physical activity at work. Eating patterns based on unprocessed and minimally processed foods and practice of physical activity are protective factors against obesity.

Key words Surveillance, Eating habits, Exercise, Obesity
\end{abstract}

Resumo O objetivo deste artigo é descrever a associação entre variação de IMC, padrões alimentares e atividade fisica entre adultos de 21 a 44 anos no período de 2007 a 2012. Estudo transversal com a base de dados do VIGITEL. Padrões alimentares foram identificados com Análise de Principais Componentes para o período de 2007 a 2012. Componentes com autovalores $>1,0$ foram retidos e cargas fatoriais superiores a $|0,3|$ foram destacadas. Para cada indivíduo, um escore foi calculado por padrão. Em seguida foi criada variável de diferença do Índice de Massa Corporal $(\triangle I M C)$. Regressão linear com desfecho $\triangle I M C$ e regressão de Poisson com desfecho obesidade foram conduzidas. Quatro padrões alimentares foram retidos: Prudente, Transição, Ocidental e Tradicional. Após ajuste multivariável, $\triangle I M C$ mostrou-se inversamente associada à prática de atividade física no lazer. Obesidade apresentou associação positiva com padrão Ocidental, assistir à televisão $\geq 3$ horas por dia e inatividade física. Obesidade mostrou-se inversamente associada com padrão Prudente, padrão Tradicional, prática de atividade fisica no lazer e prática de atividade física no trabalho. Padrões alimentares baseados em alimentos in natura e minimamente processados e prática de atividade fisica constituem fatores de proteção para a obesidade.

Palavras-chave Vigilância, Comportamento Alimentar, Exercício, Obesidade 


\section{Introdução}

Doenças crônicas não transmissíveis (DCNT) são doenças de progressão lenta que podem levar a incapacitações permanentes ou mortalidade precoce. No Brasil, 74\% dos óbitos são atribuídos às DCNT e a probabilidade de morte prematura devido a esse grupo de doenças foi estimada em $19 \%^{1}$. Os principais fatores de risco modificáveis relacionados a essas enfermidades são tabagismo, consumo excessivo de bebidas alcoólicas, inatividade física, alimentação inadequada e obesidade ${ }^{2}$.

Em 2011, o Ministério da Saúde lançou o Plano de Ações Estratégicas para o Enfrentamento das DCNT no Brasil 2011-2022. Entre as metas destacam-se: reduzir a taxa de mortalidade prematura por DCNT, deter o crescimento da obesidade em adultos, aumentar a prevalência de atividade física no lazer e aumentar o consumo de frutas e hortaliças².

A alimentação, quando adequada, é um dos fatores mais importantes para promoção da saúde e prevenção de doenças. No Brasil, os dados de consumo de alimentos considerados como marcadores de dietas de boa ou má qualidade ainda são ambivalentes quando avaliados pontualmente. Em 2013, por exemplo, o consumo regular de feijão pela população brasileira foi de $72 \%$ e o consumo recomendado de frutas e hortaliças de $37 \%{ }^{3}$. Por outro lado, no mesmo ano, a frequência de alimentos considerados como fatores de risco para DCNT, como consumo de carne ou frango com excesso de gordura foi de $37 \%$ e o consumo regular de refrigerante ou suco artificial alcançou $23 \%{ }^{4}$. A tendência do consumo desses alimentos índices, no entanto, sugere que a alimentação dos brasileiros está regredindo em qualidade. Considerando os adultos brasileiros residentes nas capitais de estado e no Distrito Federal, o consumo regular de feijão, por exemplo, reduziu de $68 \%$ em 2011 para $60 \%$ em $2017^{5}$.

$\mathrm{Na}$ outra ponta do balanço energético, temos o gasto das calorias disponíveis, entre as formas de despender calorias destacamos a atividade física que pode ser compreendida como qualquer movimento voluntário da musculatura esquelética que leva ao gasto energético. As práticas de atividade física podem ser categorizadas em diferentes domínios: ocupacionais, domésticas, deslocamento e lazer ${ }^{6}$. A recomendação da Organização Mundial da Saúde (OMS) para indivíduos adultos é de pelo menos 150 minutos semanais de atividade física leve ou moderada ou 75 minutos de atividade física vigorosa ${ }^{7}$. No Brasil, os dados de prática de atividade física sugerem padrão de baixa frequência entre os adultos. Em 2013, a frequência de adultos ativos no lazer foi de $23 \%$ e de adultos ativos no deslocamento foi de $32 \%$. Nessa mesma perspectiva, $46 \%$ dos adultos brasileiros foram considerados insuficientemente ativos e $29 \%$ assistiam à televisão mais do que 3 horas por dia ${ }^{8}$. Considerando os adultos brasileiros residentes nas capitais de estado e no Distrito Federal, houve redução da frequência de indivíduos inativos de 16\% em 2009 para $14 \%$ em 2018 e aumento da frequência de ativos no lazer de $30 \%$ para $38 \%$ no mesmo período9.

Entre adultos, valores do Índice de Massa Corporal (IMC) estão associados ao aumento do risco para doenças crônicas como diabetes, doenças cardiovasculares e neoplasias ${ }^{10}$. No Brasil, a tendência secular do estado nutricional de adultos vem sendo analisada a partir de dados de inquéritos populacionais. As prevalências de excesso de peso e de obesidade aumentaram continuamente no período de 1974-1975 a 20082009 para ambos os sexos ${ }^{11}$. Comparando dois inquéritos recentes, observa-se que a tendência observada no período anterior se manteve, a prevalência de excesso de peso aumentou de $49 \%$ em 2008-2009 para 57\% em 2013 e a prevalência de obesidade aumentou de $15 \%$ para $21 \%$ no mesmo período ${ }^{11,12}$.

No Global Burden of Disease (GBD) 2015, os fatores de risco são agrupados em comportamentais, metabólicos ou fisiológicos e ambientais. Em 2015, 39\% dos anos de vida perdidos por mortalidade prematura e anos vividos com incapacidade (Disability Adjusted Life Years - DALY) foram atribuídos aos fatores de risco avaliados conjuntamente. Em homens, a dieta inadequada contribuiu com $12 \%$ do total de DALY e foi associado a doenças cardiovasculares, diabetes e neoplasias. O segundo fator de risco para homens foi o uso de álcool e drogas, seguido de pressão arterial sistólica elevada, tabagismo, IMC elevado e glicose de jejum elevada. Em mulheres, $11 \%$ do total de DALY foram atribuídos à dieta inadequada. O segundo fator de risco para mulheres foi pressão arterial sistólica elevada, seguido de IMC elevado, glicose de jejum elevada e tabagismo ${ }^{10}$.

No Brasil ainda não dispomos de análises de porte nacional que associem padrões alimentares e atividade física com risco de excesso de peso ou obesidade com base no mesmo conjunto de dados. Essa perspectiva analítica é relevante para quantificar diretamente os riscos associados aos padrões de atividade física ou alimentares mais prevalentes no país e, estimar o conjunto de pessoas expostas a esses riscos. Dessa forma, o obje- 
tivo deste estudo foi descrever a associação entre variação de IMC, padrões alimentares e atividade física entre adultos de 21 a 44 anos no período de 2007 a 2012.

\section{Métodos}

Estudo transversal com dados do Sistema de Vigilância de Fatores de Risco e Proteção para Doenças Crônicas por Inquérito Telefônico (VIGITEL) no período de 2007 a 2012. No VIGITEL é realizada amostragem probabilística da população adulta ( $\geq 18$ anos) residente em domicílios com pelo menos uma linha telefônica fixa no ano das capitais dos 26 estados brasileiros e do Distrito Federal. O uso de fatores de expansão da amostra que igualam a composição sociodemográfica da população residente em domicílios com pelo menos uma linha telefônica fixa à população total de cada capital de estado e do Distrito Federal tornam os dados do VIGITEL representativos da população adulta residente em domicílios das capitais de estado e do Distrito Federal. Os aspectos relativos à metodologia de pesquisa do VIGITEL estão disponíveis em publicações oficiais, assim como o questionário completo do inquérito ${ }^{13}$.

De acordo com dados da PNS 2013, a prevalência de obesidade aumenta com a idade até os 65 anos e no início da vida adulta as prevalências de obesidade são menores do que nas faixas etárias entre 45 e 64 anos $^{12}$. O impacto do aumento da massa corporal sobre a qualidade de vida e a sua associação com morbidades pode influenciar as escolhas alimentares ${ }^{14}$. A amostra selecionada neste estudo é composta por indivíduos adultos de 18 a 44 anos de idade com o objetivo de minimizar o impacto de mudanças em padrões alimentares em virtude do estado nutricional atual. Mulheres grávidas ou que não sabiam se estavam grávidas foram excluídas da análise. Neste estudo foram incluídos 167.761 indivíduos, de 2007 foram incluídos 31.291, de 2008 foram incluídos 30.051 , de 2009 foram incluídos 29.310, de 2010 foram incluídos 28.371, de 2011 foram incluídos 27.133 e de 2012 foram incluídos 21.605. A amostra selecionada para as análises de regressão linear e regressão de Poisson é composta por indivíduos de 21 a 44 anos, pois a variável peso aos 20 anos foi coletada apenas para indivíduos com mais de 20 anos. Por isso, nas análises de associação foram incluídos 148.748 indivíduos, de 2007 foram incluídos 27.597, de 2008 foram incluídos 26.545, de 2009 foram incluídos 25.991, de 2010 foram incluídos 25.280, de 2011 foram incluídos
24.089 e de 2012 foram incluídos 19.246. O período selecionado neste estudo é de 2007 a 2012, o ano 2006 não foi incluído, pois algumas variáveis de consumo alimentar não possuíam a mesma codificação utilizada a partir de 2007. O período prolongou-se apenas até 2012, pois a partir de 2013, o VIGITEL descontinuou a coleta da variável peso aos 20 anos.

As variáveis sociodemográficas utilizadas foram faixa etária, sexo, estado civil e escolaridade. As variáveis comportamentais utilizadas foram fumante, ex-fumante e consumo abusivo de álcool. As variáveis relacionadas ao estado nutricional utilizadas foram IMC aos 20 anos categórica $\left(\mathrm{IMC}_{20}\right)$, obesidade e $\triangle \mathrm{IMC}$ (IMC atual - IMC aos 20 anos). As variáveis de consumo alimentar utilizadas foram: consumo semanal de hortaliças, de hortaliças cozidas, de hortaliças cruas, de feijão, de carne vermelha, de frango, de frutas, de refrigerante ou suco artificial, de leite, consumo diário de hortaliças e consumo de gordura. As variáveis de atividade física (AF) utilizadas foram frequência semanal de prática de AF no lazer, AF no trabalho, estimativa de gasto energético por $\mathrm{AF}$ no lazer, inatividade física e assistir à televisão $\geq 3$ horas por dia. A variável IMC aos 20 anos $\left(\mathrm{IMC}_{20}\right)$ e a obesidade foram classificadas de acordo com o proposto pela $\mathrm{OMS}^{15}$. O gasto energético foi estimado por tipo de atividade física segundo valores de equivalente metabólico extraídos do Compêndio de Atividade Física ${ }^{16}$.

As variáveis estado civil, massa corporal, IMC atual, IMC aos 20 anos e estimativa de gasto energético por AF foram imputadas por Predictive Mean Matching (PMM) que envolve a especificação de modelo para dados faltantes a partir de valores observados na amostra ${ }^{17}$. A PMM combina regressão linear para obter predições lineares e seleção aleatória de valor para imputação entre vizinhos mais próximos ${ }^{18}$. Valores observados próximos ao valor predito são selecionados como o pool doador. O pool doador é fixado contendo $k$ candidatos doadores ${ }^{17}$, em nosso estudo $k$ foi fixado em 5 . Um desses valores foi aleatoriamente selecionado e o seu valor foi copiado no lugar do dado faltante. As variáveis utilizadas no modelo para realizar a imputação das variáveis estado civil, IMC atual, IMC aos 20 anos e massa corporal foram sexo, idade e cidade. As variáveis utilizadas para realizar a imputação de estimativa de gasto energético por AF foram prática de AF, frequência semanal da prática de AF no lazer, sexo, idade e cidade. A variável IMC aos 20 anos apresentou o maior percentual de imputação $(25,7 \%)$. Neste estudo preservou-se o tamanho da amostra e a 
sua representatividade ao utilizar as codificações propostas pelo VIGITEL e imputação por meio da PMM.

Os padrões alimentares foram identificados com a técnica multivariada Análise de Componentes Principais (ACP). A ACP é uma análise do tipo fatorial que reduz os dados em padrões baseados nas correlações entre as varáveis ${ }^{19}$. O primeiro componente principal corresponde à direção de maior variância. Os demais componentes são ortogonais aos componentes anteriores e modelam a direção de maior variância do espaço não modelado pelos componentes anteriores ${ }^{20}$. As duas propriedades básicas da ACP são os autovalores e os autovetores. Os autovalores indicam o total da variância de cada componente ${ }^{21}$. As cargas fatoriais (CF) definem os autovetores que são os componentes principais ${ }^{20}$. Carga fatorial positiva indica impacto positivo da variável no componente e carga fatorial negativa indica impacto negativo ${ }^{21}$.

As variáveis de consumo alimentar utilizadas na ACP foram: consumo semanal de hortaliças, de hortaliças cozidas, de hortaliças cruas, de feijão, de carne vermelha, de frango, de frutas, de refrigerante ou suco artificial, de leite, consumo diário de hortaliças e consumo de gordura. As variáveis de frequência semanal possuem a mesma codificação: 0 - nunca; 0,5 - quase nunca; 1,5 - 1 a 2 dias; 3,5 - 3 a 4 dias; 5,5 - 5 a 6 dias; 7 - todos os dias. Consumo diário de hortaliças corresponde ao somatório das respostas dadas às questões "Num dia comum, o(a) Senhor(a) come esse tipo de salada?" e "Num dia comum, o(a) Senhor(a) come verdura ou legume cozido?", se o indivíduo comia no almoço ou jantar a essas questões foi atribuído o número 1 e se comia nas duas refeições foi atribuído o número 2. A variável dicotômica consumo de gordura visível refere-se as respostas às questões "Quando o(a) Senhor(a) come carne vermelha com gordura, o(a) Senhor(a) costuma:" "Quando o(a) Senhor(a) come frango com pele, o(a) Senhor(a) costuma:", foram consideradas as respostas "comer com a gordura" e "comer com a pele" para atribuir o valor 1 a essa variável.

$\mathrm{Na} \mathrm{ACP}$ foram retidos os componentes com autovalores $>1,0$ conforme a Regra de Kaiser e foram destacadas cargas fatoriais superiores a $|0,3|^{21}$. O teste de Kaiser-Meyer-Olkin (KMO) foi utilizado para avaliar a adequação dos padrões formados ao conjunto de dados ${ }^{19}$. A estatística KMO varia entre 0 e 1: valores abaixo de 0,5 são considerados inaceitáveis, entre 0,50 e 0,59 ruins, entre 0,60 e 0,69 medíocres, entre 0,70 e 0,79 ra- zoáveis, entre 0,80 e 0,89 bons e entre 0,90 e 1,0 excelentes ${ }^{22}$.

Após a identificação dos padrões, para cada padrão foram gerados escores padronizados com média zero; portanto, cada indivíduo tem escores em todos os padrões. A análise foi realizada para o conjunto de anos de monitoramento do VIGITEL 2007-2012. Os componentes retidos no conjunto 2007-2012 apresentam correlações superiores a 0,90 com os padrões retidos em cada ano separadamente. Após a extração não foi aplicada nenhum tipo de rotação, pois, os efeitos da rotação são únicos para cada matriz de dados.

Neste estudo foram analisados fatores associados às variações de IMC e fatores associados à obesidade. No primeiro modelo foi utilizada regressão linear com desfecho $\triangle \mathrm{IMC}$ e no segundo modelo foi utilizada regressão de Poisson com desfecho obesidade. Nas análises foram utilizadas as variáveis de padrões alimentares, as variáveis relacionadas à prática de atividade física, as variáveis sociodemográficas, o estado nutricional aos 20 anos e as variáveis comportamentais. $\mathrm{Na}$ análise de regressão linear foram utilizadas as opções eform ( ) e noconstant do Stata. Inicialmente, foi necessário transformar a variável $\triangle \mathrm{IMC}$ na escala logarítmica e gerar uma variável constante que informa a média geométrica no modelo de regressão. A variável $\triangle \mathrm{IMC}$ possui números negativos e positivos, no conjunto dos números reais não existe logaritmo de 0 e de número negativo. Por isso, optou-se por somar uma constante à variável $\triangle \mathrm{IMC}$ e assim realizar a transformação. Nesta análise, os parâmetros das variáveis independentes contínuas são interpretados como uma razão de $\mathrm{Y}$ por unidade de mudança em $\mathrm{X}$, assumindo uma relação exponencial ${ }^{23}$. $\mathrm{Na}$ análise com desfecho $\triangle \mathrm{IMC}$, objetivou-se analisar a associação entre o incremento ou a redução de IMC, os padrões alimentares e a prática de atividade física. Em relação a análise com desfecho obesidade, dado que o indivíduo apresenta obesidade atualmente, objetivou-se analisar como estão associados às variáveis relacionadas à prática de atividade física e os padrões alimentares a obesidade. Em função do tamanho da amostra, p-valores não foram apresentados. Nas duas análises utilizou-se o módulo Survey do Stata que considera os efeitos da amostragem. Todas as análises foram realizadas usando o software Stata ${ }^{\circledR}$.

Os dados deste estudo foram coletados pelo VIGITEL. O consentimento livre e esclarecido foi obtido oralmente no momento do contato telefônico com os entrevistados. O projeto VIGITEL foi aprovado pela Comissão Nacional de Ética em 
Pesquisa para Seres Humanos do Ministério da Saúde $^{13}$. O presente estudo foi apreciado e aprovado pelo Comitê de Ética em Pesquisa da Faculdade de Saúde Pública da Universidade de São Paulo.

\section{Resultados}

Os padrões alimentares foram estimados para o conjunto de dados de 2007 a 2012 e o valor do KMO foi razoável $(0,7301)$. Os quatro componentes retidos em conjunto explicaram 55,9\% da variabilidade total dos dados. Os componentes principais ou padrões alimentares foram rotulados de acordo com as características observadas na ACP. Na Tabela 1 apresentam-se os padrões e suas respectivas cargas fatoriais (CF), autovalores e percentual da variância explicada.

O padrão Prudente se caracterizou pelas variáveis com $\mathrm{CF}+$ consumo semanal de hortaliças, de hortaliças cruas, de hortaliças cozidas e consumo diário de hortaliças, esse padrão explicou 23,8\% da variabilidade total dos dados. O padrão Transição se caracterizou pelas variáveis com CF+ consumo semanal de carne vermelha e de refrigerante ou suco artificial e consumo de gordura, e com CF- consumo semanal de frango, esse padrão explicou $13,1 \%$ da variabilidade total dos dados. O padrão Ocidental se caracterizou pelas variáveis com $\mathrm{CF}+$ consumo semanal de frango e de refrigerante ou suco artificial, e com CF- consumo semanal de leite, esse padrão ex- plicou 9,8\% da variabilidade total dos dados. O padrão Tradicional se caracterizou pelas variáveis com CF+ consumo semanal de feijão, de frango e de leite, esse padrão explicou 9,2\% da variabilidade total dos dados.

$\mathrm{Na}$ Tabela 2 é apresentada a análise multivariável modelada por regressão linear com desfecho $\triangle I M C$. A variável $\triangle I M C$ apresentou associação positiva com casado ou união estável, separado ou divorciado, maior idade, baixo peso aos 20 anos e consumo abusivo de bebidas alcoólicas. A variável $\triangle \mathrm{IMC}$ mostrou-se inversamente associada a 12 ou mais anos de escolaridade, pré -obesidade aos 20 anos, obesidade aos 20 anos e frequência semanal de prática de atividade física no lazer. Assumindo uma relação exponencial, para cada aumento de uma unidade na prática de atividade física no lazer, houve redução de $1 \%$ na média geométrica de $\triangle \mathrm{IMC}$.

Na Tabela 3 é apresentada a análise multivariável modelada por regressão de Poisson com desfecho obesidade. A variável obesidade apresentou associação positiva com sexo feminino, maior idade, casado ou união estável, pré-obesidade aos 20 anos, obesidade aos 20 anos, consumo abusivo de bebidas alcoólicas, ex-fumante, padrão Ocidental, assistir à televisão $\geq 3$ horas por dia e inatividade física. A variável obesidade mostrou-se inversamente associada a maior escolaridade, baixo peso aos 20 anos, padrão Prudente, padrão Tradicional, frequência semanal de prática de atividade física no lazer e prática de atividade física no trabalho.

Tabela 1. Descrição dos padrões alimentares de indivíduos de 18 a 44 anos. VIGITEL, 2007-2012.

\begin{tabular}{lcccc}
\hline \multirow{2}{*}{ Variáveis } & \multicolumn{2}{c}{ Cargas Fatoriais } \\
\cline { 2 - 5 } Feijão & Prudente & Transição & Ocidental & Tradicional \\
Hortaliças & 0,0308 & 0,2522 & $-0,0816$ & $\mathbf{0 , 8 0 9 5}$ \\
Hortaliças cozidas & $\mathbf{0 , 5 0 5 6}$ & 0,1235 & 0,0901 & $-0,0958$ \\
Hortaliças cruas & 0,4620 & 0,1655 & 0,0566 & $-0,1096$ \\
Consumo diário de hortaliças & $\mathbf{0 , 4 3 3 7}$ & 0,0604 & 0,1478 & 0,0198 \\
Frutas & $\mathbf{0 , 4 3 9 9}$ & 0,1723 & 0,1038 & 0,0501 \\
Carne vermelha & 0,2841 & $-0,1703$ & $-0,2972$ & $-0,0359$ \\
Frango & $-0,0636$ & $\mathbf{0 , 5 8 5 9}$ & $-0,2933$ & $-0,0579$ \\
Gordura & 0,0615 & $\mathbf{- 0 , 3 7 1 2}$ & $\mathbf{0 , 5 5 0 7}$ & $\mathbf{0 , 4 1 0 2}$ \\
Refrigerante ou suco artificial & $-0,1471$ & $\mathbf{0 , 4 3 6 6}$ & 0,2324 & 0,1388 \\
Leite & $-0,1636$ & $\mathbf{0 , 3 7 6 2}$ & $\mathbf{0 , 3 2 4 7}$ & $-0,0441$ \\
Autovalores & 0,1069 & $-0,1349$ & $\mathbf{- 0 , 5 5 8 8}$ & $\mathbf{0 , 3 5 5 6}$ \\
\% Variância & 2,6132 & 1,4416 & 1,0767 & 1,0161 \\
\% Variância acumulada & $23,8 \%$ & $13,1 \%$ & $9,8 \%$ & $9,2 \%$ \\
\hline Nat Cargas & $23,8 \%$ & $36,9 \%$ & $46,7 \%$ & $55,9 \%$ \\
\hline
\end{tabular}

Nota: Cargas fatoriais superiores a $|0,3|$ foram destacadas. 
Tabela 2. Análise multivariável dos fatores associados às variações de IMC entre indivíduos de 21 a 44 anos. VIGITEL, 2007-2012.

\begin{tabular}{|c|c|c|c|}
\hline Variáveis & Razão & Erro padrão linearizado & IC95\%* \\
\hline \multicolumn{4}{|l|}{ Sexo } \\
\hline Masculino & 1 & & \\
\hline Feminino & 1,00 & $<0,01$ & 1,$00 ; 1,01$ \\
\hline \multicolumn{4}{|l|}{ Faixa etária } \\
\hline 21 a 24 anos & 1 & & \\
\hline 25 a 34 anos & 1,03 & $<0,01$ & 1,$03 ; 1,03$ \\
\hline 35 a 44 anos & 1,06 & $<0,01$ & 1,$05 ; 1,06$ \\
\hline \multicolumn{4}{|l|}{ Estado civil } \\
\hline Solteiro & 1 & & \\
\hline Casado ou união estável & 1,02 & $<0,01$ & 1,$02 ; 1,02$ \\
\hline Viúvo & 1,03 & 0,01 & 1,$00 ; 1,05$ \\
\hline Separado ou divorciado & 1,01 & $<0,01$ & 1,$01 ; 1,01$ \\
\hline \multicolumn{4}{|l|}{ Escolaridade } \\
\hline 0 a 8 anos & 1 & & \\
\hline 9 a 11 anos & 1,00 & $<0,01$ & 0,$99 ; 1,00$ \\
\hline 12 ou mais anos & 0,99 & $<0,01$ & 0,$99 ; 0,99$ \\
\hline \multicolumn{4}{|l|}{$\mathrm{IMC}_{20}$} \\
\hline Adequado & 1 & & \\
\hline Baixo peso & 1,05 & $<0,01$ & 1,$04 ; 1,05$ \\
\hline Pré-obesidade & 0,95 & $<0,01$ & 0,$95 ; 0,96$ \\
\hline Obesidade & 0,82 & 0,01 & 0,$80 ; 0,83$ \\
\hline \multicolumn{4}{|l|}{ Consumo abusivo de álcool } \\
\hline Não & 1 & & \\
\hline Sim & 1,01 & $<0,01$ & 1,$01 ; 1,01$ \\
\hline \multicolumn{4}{|l|}{ Fumante } \\
\hline Não & 1 & & \\
\hline Sim & 0,99 & $<0,01$ & 0,$98 ; 1,00$ \\
\hline \multicolumn{4}{|l|}{ Ex-fumante } \\
\hline Não & 1 & & \\
\hline Sim & 1,01 & $<0,01$ & 1,$00 ; 1,01$ \\
\hline Prudente & 1,00 & $<0,01$ & 1,$00 ; 1,00$ \\
\hline Transição & 1,00 & $<0,01$ & 1,$00 ; 1,00$ \\
\hline Ocidental & 1,00 & $<0,01$ & 1,$00 ; 1,00$ \\
\hline Tradicional & 1,00 & $<0,01$ & 1,$00 ; 1,00$ \\
\hline Frequência semanal de prática $\mathrm{AF}$ & 0,99 & $<0,01$ & 0,$99 ; 0,99$ \\
\hline Estimativa de gasto energético por $\mathrm{AF}$ & 1,00 & $<0,01$ & 1,$00 ; 1,00$ \\
\hline \multicolumn{4}{|l|}{ AF no trabalho } \\
\hline Não & 1 & & \\
\hline Sim & 1,00 & $<0,01$ & 0,$99 ; 1,00$ \\
\hline \multicolumn{4}{|l|}{ Assistir à televisão $\geq 3$ horas por dia } \\
\hline Não & 1 & & \\
\hline Sim & 1,01 & $<0,01$ & 1,$00 ; 1,01$ \\
\hline \multicolumn{4}{|l|}{ Inativo } \\
\hline Não & 1 & & \\
\hline Sim & 1,01 & $<0,01$ & 1,$00 ; 1,01$ \\
\hline Média geométrica & 1,10 & $<0,01$ & 1,$10 ; 1,10$ \\
\hline
\end{tabular}

Fonte: Elaborado pelos autores. 
Tabela 3. Análise multivariável dos fatores associados à obesidade entre indivíduos de 21 a 44 anos. VIGITEL, 2007-2012.

\begin{tabular}{|c|c|c|c|}
\hline Variáveis & Razão de prevalências & Erro padrão linearizado & IC95\%* \\
\hline \multicolumn{4}{|l|}{ Sexo } \\
\hline Masculino & 1 & & \\
\hline Feminino & 1,16 & 0,03 & 1,$11 ; 1,22$ \\
\hline \multicolumn{4}{|l|}{ Faixa etária } \\
\hline 21 a 24 anos & 1 & & \\
\hline 25 a 34 anos & 1,96 & 0,12 & 1,$73 ; 2,22$ \\
\hline 35 a 44 anos & 2,74 & 0,21 & 2,$35 ; 3,20$ \\
\hline \multicolumn{4}{|l|}{ Estado civil } \\
\hline Solteiro & 1 & & \\
\hline Casado ou união estável & 1,30 & 0,04 & 1,$23 ; 1,38$ \\
\hline Viúvo & 1,24 & 0,14 & 0,$98 ; 1,55$ \\
\hline Separado ou divorciado & 1,08 & 0,04 & 1,$00 ; 1,17$ \\
\hline \multicolumn{4}{|l|}{ Escolaridade } \\
\hline 0 a 8 anos & 1 & & \\
\hline 9 a 11 anos & 0,92 & 0,03 & 0,$86 ; 0,97$ \\
\hline 12 ou mais anos & 0,80 & 0,02 & 0,$76 ; 0,84$ \\
\hline \multicolumn{4}{|l|}{$\mathrm{IMC}_{20}$} \\
\hline Adequado & 1 & & \\
\hline Baixo peso & 0,50 & 0,03 & 0,$44 ; 0,56$ \\
\hline Pré-obesidade & 3,14 & 0,09 & 2,$96 ; 3,32$ \\
\hline Obesidade & 5,11 & 0,43 & 4,$29 ; 6,09$ \\
\hline \multicolumn{4}{|l|}{ Consumo abusivo de álcool } \\
\hline Não & 1 & & \\
\hline Sim & 1,12 & 0,04 & 1,$04 ; 1,20$ \\
\hline \multicolumn{4}{|l|}{ Fumante } \\
\hline Não & 1 & & \\
\hline Sim & 0,90 & 0,05 & 0,$81 ; 1,01$ \\
\hline \multicolumn{4}{|l|}{ Ex-fumante } \\
\hline Não & 1 & & \\
\hline Sim & 1,15 & 0,04 & 1,$07 ; 1,24$ \\
\hline Prudente & 0,95 & 0,01 & 0,$94 ; 0,96$ \\
\hline Transição & 1,02 & 0,01 & 1,$00 ; 1,04$ \\
\hline Ocidental & 1,09 & 0,02 & 1,$04 ; 1,13$ \\
\hline Tradicional & 0,95 & 0,01 & 0,$93 ; 0,97$ \\
\hline Frequência semanal de prática AF & 0,82 & 0,01 & 0,$79 ; 0,85$ \\
\hline Estimativa de gasto energético por AF & 1,00 & $<0,01$ & 1,$00 ; 1,00$ \\
\hline \multicolumn{4}{|l|}{ AF no trabalho } \\
\hline Não & 1 & & \\
\hline $\operatorname{Sim}$ & 0,91 & 0,03 & 0,$85 ; 0,97$ \\
\hline \multicolumn{4}{|l|}{ Assistir à televisão $\geq 3$ horas por dia } \\
\hline Não & 1 & & \\
\hline Sim & 1,14 & 0,02 & 1,$10 ; 1,19$ \\
\hline \multicolumn{4}{|l|}{ Inativo } \\
\hline Não & 1 & & \\
\hline Sim & 1,17 & 0,04 & 1,$09 ; 1,25$ \\
\hline
\end{tabular}

Legenda: Prudente: $\mathrm{CF}+$ consumo semanal e diário de hortaliças, hortaliças cruas e hortaliças cozidas; Transição: $\mathrm{CF}+$ carne vermelha, gordura e refrigerante ou suco artificial, CF- frango; Ocidental: CF+ frango e refrigerante ou suco artificial, CF- leite; Tradicional: CF+ feijão, frango e leite; ${ }^{\star} \mathrm{IC} 95 \%$ : Intervalo de $95 \%$ de confiança.

Fonte: Elaborado pelos autores. 


\section{Discussão}

Nossos resultados indicam que: a) os padrões alimentares Prudente e Tradicional estavam inversamente associados à obesidade, b) o padrão Ocidental apresentou associação positiva com obesidade, c) prática de atividade física no lazer estava inversamente associada à $\triangle \mathrm{IMC}$ e à obesidade e prática de atividade física no trabalho estava inversamente associada à obesidade, d) assistir à televisão por 3 ou mais horas por dia e inatividade física apresentaram associação positiva com obesidade, e) escolaridade elevada apresentou-se como fator de proteção para o incremento de IMC após os 20 anos de idade e para a obesidade, f) $\triangle \mathrm{IMC}$ apresentou associação inversa com excesso de peso aos 20 anos, g) obesidade apresentou associação positiva com excesso de peso aos 20 anos.

Esses achados estão, em grande parte, alinhados com resultados observados em outros estudos. No Inquérito de Saúde no Município de São Paulo (ISA-Capital) realizado em 2008, o padrão Tradicional (CF+ arroz e feijão e CF- massa) foi associado com menor IMC entre indivíduos insuficientemente ativos ${ }^{24}$. Na Argentina, observou-se associação positiva entre o padrão Ocidental ( $\mathrm{CF}+$ carnes e ovos, carnes processadas e bebidas alcoólicas) e obesidade ${ }^{25}$. Em Québec no Canadá, observou-se associação positiva entre o padrão Ocidental (CF+ grãos refinados, batatas fritas, carnes vermelhas, condimentos, carnes processadas, refrigerantes, pizza, lanches, batatas, sobremesas e cerveja e CF- leguminosas, frutas e vegetais) e obesidade; e associação inversa entre o padrão Prudente $(\mathrm{CF}+$ gordura não hidrogenada, vegetais, ovos, peixes e outros frutos do mar, vinho, café, leite e derivados e grãos integrais) e obesidade ${ }^{26}$.

Análise realizada com dados do Sistema $\mathrm{Mu}$ nicipal de Monitoramento de Fatores de Risco para Doenças Crônicas Não Transmissíveis (Simtel) no ano de 2005 em Florianópolis-SC definiu sedentarismo de maneira similar a variável inatividade física do VIGITEL. Na análise, sedentarismo mostrou-se associado ao aumento de $\mathrm{IMC}^{27}$. Com base em dados de adultos da National Health and Nutrition Survey analisou-se a associação entre atividade física e obesidade. O estudo utilizou três medidas: atividade física moderada-vigorosa e tempo sedentário total a partir dos dados do acelerômetro e tempo de assistir à televisão referido. Observou-se associação positiva entre moderado e baixo tempo de atividade física moderada-vigorosa com obesidade em ambos os sexos e associação positiva entre moderado e alto tempo de assistir à televisão com obesidade em homens e associação positiva entre alto tempo de assistir à televisão com obesidade em mulheres ${ }^{28}$.

No presente estudo, maior nível de escolaridade é fator protetor para incremento de IMC após os 20 anos de idade e para obesidade. Em estudo com dados da POF 2008-2009, indivíduos com 0 a 5 anos de escolaridade (RP: 1,03) e indivíduos com 6 a 9 anos de escolaridade (RP: 1,07) tiveram maior risco de excesso de peso do que indivíduos com 10 ou mais anos de escolaridade ${ }^{29}$. Em Belo Horizonte-MG, o IMC e a obesidade se associaram inversamente à escolaridade entre mulheres $^{30}$. Em Florianópolis-SC, observou-se associação entre aumento de IMC e menor escolaridade entre mulheres ${ }^{27}$.

A escolaridade como um marcador de desigualdade pode explicar parcialmente os nossos resultados. Para explicar essas diferenças podemos recorrer ao trabalho conduzido por Bilger et al. ${ }^{31}$ com utilização de dados dos inquéritos nacionais dos Estados Unidos do período de 19712012, no qual demonstrou-se que a desigualdade social em relação ao status obesidade reduziu no período. Os autores sugerem que fatores ambientais passaram a ser mais significativos na determinação da obesidade do que características individuais. Por outro lado, ao considerar a profundidade e a gravidade da obesidade, as desigualdades sociais foram mais expressivas, portanto, políticas públicas direcionadas a dupla carga de pobreza e condições relacionadas a obesidade são necessárias.

Em nosso estudo, padrão alimentar marcado positivamente pela presença de alimento ultraprocessado (refrigerante ou sucos artificiais) mostrou-se associado a obesidade. A adesão a este padrão foi maior entre indivíduos com até 8 anos de escolaridade, enquanto que indivíduos com 12 ou mais anos de escolaridade tiveram maior adesão ao padrão Prudente ${ }^{32}$. A associação entre o consumo de alimentos ultraprocessados e desfechos relacionados a saúde já foram previamente descritos na literatura. E Baraldi et al. ${ }^{33}$ demonstraram que o consumo de alimentos ultraprocessados foi maior entre indivíduos mais jovens, menos escolarizados e de menor renda.

Em países submetidos a fortes vetores pró -obesidade, como o Brasi ${ }^{34}$, mesmo quando indivíduos com excesso de peso aos 20 anos reduzem seu IMC para faixas saudáveis, eles são substituídos por aqueles que ganham peso na idade adulta, o que faz com que o nível de obesidade se mantenha inalterado ou aumente. No estudo de Flo- 
rianópolis, observou-se associação inversa entre o aumento do IMC e o IMC aos 20 anos em ambos os sexos, e aumento da prevalência de excesso de peso $^{27}$. Em estudo com dados do VIGITEL estimou-se a incidência de excesso de peso entre homens que aos 20 anos apresentavam baixo peso ou peso normal em $40 \%$ e entre mulheres em $30 \%$. A persistência da obesidade foi estimada em $65 \%$ entre homens e em $47 \%$ entre mulheres ${ }^{35}$.

Nossas análises apresentam alguns aspectos que merecem comentários detalhados. Em desenhos transversais as variáveis de exposição e desfecho são coletadas ao mesmo tempo o que favorece análises de associação e inviabiliza as de causalidade. Em nossa análise, a única variável que remete a período anterior é o peso aos 20 anos, utilizada no cálculo da variável $\triangle \mathrm{IMC}$. Potencialmente temos aqui uma vulnerabilidade que é o viés de memória dessa informação. Em outra análise dos dados do VIGITEL já se pôde evidenciar que o perfil de suas informações antropométricas está bem associado ao perfil dos dados de inquéritos com mensuração antropométrica ${ }^{36}$. Para verificar a plausibilidade da informação do peso aos 20 anos comparamos, então, o valor médio dessa informação com dados dos inquéritos anteriores do VIGITEL. Por exemplo, a coorte de 25 anos do VIGITEL-2011 foi comparada aos dados antropométricos correntes do VIGITEL-2006 e, assim, sucessivamente (dados não apresentados aqui). Os resultados mostraram a consistência da informação do peso recordado em ambos os sexos. Além disso, o aumento do excesso de peso diferenciado entre os sexos nas idades iniciais da vida adulta nas duas séries de informação (recordada e contemporânea) sugere a consistência satisfatória dessa informação.

As variáveis de alimentação coletadas pelo VIGITEL são marcadores de consumo alimentar. Monteiro et al. ${ }^{37}$, em estudo para avaliar a validade dos indicadores do consumo de alimentos e bebidas por inquérito telefônico indicou boa reprodutibilidade e adequada validade desses in- dicadores. Outra possível limitação deste estudo está relacionada ao uso exclusivo pelo VIGITEL do cadastro de linhas telefônicas fixas. Em estudo conduzido por Bernal et al. ${ }^{38}$, ao estudar o efeito da inclusão de entrevistas por telefone celular ao VIGITEL, sugeriram a inclusão de subamostra com somente telefone celular, especialmente nas capitais das regiões Norte e Nordeste.

Em nossas análises, tanto o padrão alimentar Ocidental quanto o hábito de assistir à televisão $\geq 3$ horas por dia e a inatividade física se mostraram associados à obesidade. Os comportamentos foram medidos individualmente, porém, esses comportamentos são influenciados por diversos fatores como ambientais, culturais, psicológicos e sociais. Por exemplo, mudanças no sistema agrário e o crescimento da indústria promoveram as alterações de padrões alimentares observadas nas últimas décadas como o aumento do consumo de carboidratos refinados, óleos comestíveis, bebidas açucaradas e alimentos de origem animal e redução da ingestão de leguminosas, frutas, legumes e verduras ${ }^{39,40}$. As modificações decorrentes do processo de urbanização e industrialização promoveram alterações significativas nos padrões de prática de atividade física, acarretando em aumento da frequência do comportamento sedentário, e, portanto, redução de gasto energético. Por exemplo, a atividade física no lazer que deveria ser preponderante tem sido substituída por atividades de baixo gasto energético como o uso intensivo de computadores, aparelhos de telefonia móvel e televisão ${ }^{39-41}$.

Os dados sugerem que há fortes vetores favoráveis às tendências de ganho de peso e aumento da obesidade no Brasil e que é razoável supor que as metas originais do Plano de Ações Estratégicas para o Enfrentamento das DCNT no Brasil 20112022 dificilmente se concretizarão mediante tais tendências. Assim, é recomendável a realização de avaliação intermediária que permita recalibrar metas, processos ou ações previstas para controle da obesidade no Brasil. 


\section{Colaboradores}

IKS Santos: concepção do estudo, revisão da literatura, análise e interpretação dos dados, elaboração da primeira versão do artigo, revisão crítica do texto, aprovação da versão final do artigo. WL Conde: concepção do estudo, análise e interpretação dos dados, revisão crítica do texto, aprovação da versão final do artigo.

\section{Referências}

1. World Health Organization (WHO). Noncommunicable Diseases: Progress Monitor 2015. Genebra: WHO; 2015.

2. Brasil. Ministério da Saúde (MS). Plano de ações estratégicas para o enfrentamento das doenças crônicas não transmissiveis (DCNT) no Brasil 2011-2022. Brasília: MS; 2011.

3. Jaime PC, Stopa SR, Oliveira TP, Vieira ML, Szwarcwald CL, Malta DC. Prevalência e distribuição sociodemográfica de marcadores de alimentação saudável, Pesquisa Nacional de Saúde, Brasil 2013. Epidemiol Serv Saude 2015; 24:267-276.

4. Claro RM, Santos MAS, Oliveira TP, Pereira CA, Szwarcwald CL, Malta DC. Consumo de alimentos não saudáveis relacionados a doenças crônicas não transmissíveis no Brasil: Pesquisa Nacional de Saúde, 2013. Epidemiol Serv Saude 2015; 24:257-265.

5. Brasil. Ministério da Saúde (MS). VIGITEL Brasil 2017. Vigilância de fatores de risco e proteção para doenças crônicas por inquérito telefônico. Estimativas sobre frequência e distribuição sociodemográfica de fatores de risco e proteção para doenças crônicas nas capitais dos 26 estados brasileiros e no Distrito Federal em 2017. Brasília: MS; 2018.

6. Caspersen CJ, Powell KE, Christenson GM. Physical activity, exercise, and physical fitness: definitions and distinctions for health-related research. Public Health Reports 1985; 100(2):126-131.

7. World Health Organization (WHO). Global recommendations on physical activity for health. Genebra: WHO; 2010.

8. Mielke GI, Hallal PC, Rodrigues GBA, Szwarcwald CL, Santos FV, Malta DC. Prática de atividade física e hábito de assistir à televisão entre adultos no Brasil: Pesquisa Nacional de Saúde 2013. Epidemiol Serv Saude 2015; 24:277-286.

9. Brasil. Ministério da Saúde (MS). VIGITEL Brasil 2018. Vigilância de fatores de risco e proteção para doenças crônicas por inquérito telefônico. Estimativas sobre frequência e distribuição sociodemográfica de fatores de risco e proteção para doenças crônicas nas capitais dos 26 estados brasileiros e no Distrito Federal em 2018. Brasília: MS; 2019.

10. Malta DC, Felisbino-Mendes MS, Machado ÍE, Passos VMA, Abreu DMX, Ishitani LH, Velásquez-Meléndez G, Carneiro M, Mooney M, Naghavi M. Fatores de risco relacionados à carga global de doença do Brasil e Unidades Federadas, 2015. Rev Bras Epidemiol 2017; 20:217-232.

11. Instituto Brasileiro de Geografia e Estatística (IBGE). Pesquisa de Orçamentos Familiares 2008-2009. Antropometria e estado nutricional de crianças, adolescentes e adultos no Brasil. Rio de Janeiro: IBGE; 2010.

12. Instituto Brasileiro de Geografia e Estatística (IBGE). Pesquisa Nacional de Saúde: 2013: Ciclos de vida: Brasil e grandes regiões. Rio de Janeiro: IBGE; 2015.

13. Brasil. Ministério da Saúde (MS). VIGITEL Brasil 2012. Vigilância de fatores de risco e proteção para doenças crônicas por inquérito telefônico. Estimativas sobre frequência e distribuição sociodemográfica de fatores de risco e proteção para doenças crônicas nas capitais dos 26 estados brasileiros e no Distrito Federal em 2012. Brasília: MS; 2013. 
14. Fernandes DPS, Duarte MSL, Pessoa MC, Franceschini SCC, Ribeiro AQ. Evaluation of diet quality of the elderly and associated factors. Arch Gerontol Geriatr 2017; 72:174-180.

15. World Health Organization (WHO). Obesity: Preventing and managing the global epidemic. Genebra: WHO; 2000.

16. Ainsworth B, Haskell WL, Herrmann S, Meckes N, Bassett D, Tudor-Locke C, Greer JL, Vezina J, Whitt-Glover MC, Leon AS. 2011 Compendium of Physical Activities: A Second Update of Codes and MET Values. Med Sci Sports Exerc 2011; 43(8):1575-1581.

17. Morris TP, White IR, Royston P. Tuning multiple imputation by predictive mean matching and local residual draws. BMC Med Res Methodol 2014; 14(1):75.

18. StataCorp. MI Impute PMM - Impute using predictive mean matching [Internet]. [acessado 2017 ago 20]. Disponível em: http://www.stata.com/manuals13/mimiimputepmm.pdf.

19. Olinto MTA. Padrões Alimentares. In: Epidemiologia Nutricional. $20^{\mathrm{a}}$ ed. Rio de Janeiro: Fiocruz/Atheneu; 2007. p. 213-225.

20. Lyra WS, Silva EC, Araújo MCU, Fragoso WD, Veras G. Classificação periódica: um exemplo didático para ensinar análise de componentes principais. Quim Nova 2010; 33:1594-1597.

21. Jolliffe IT. Principal Component Analysis. $2^{\mathrm{a}}$ ed. New York: Springer; 2002.

22. Kaiser HF. An index of factorial simplicity. Psychometrika 1974; 39(1):31-36.

23. Newson R. Stata tip 1: the eform () option of regress. Stata J 2001; 1(1):1.

24. Santos RO, Vieira DAS, Miranda AAM, Fisberg RM, Marchioni DM, Baltar VT. The traditional lunch pattern is inversely correlated with body mass index in a population-based study in Brazil. BMC Public Health 2018; 18:33.

25. Pou SA, Pilar Díaz M, De La Quintana AG, Forte CA, Aballay LR. Identification of dietary patterns in urban population of Argentina: study on diet-obesity relation in population-based prevalence study. Nutr Res Pract 2016; 10(6):616-622.

26. Paradis A-M, Godin G, Pérusse L, Vohl M-C. Associations between dietary patterns and obesity phenotypes. Int J Obes 2009; 33:1419.

27. Coelho MSPH, Assis MAA, Moura EC. Aumento do índice de massa corporal após os 20 anos de idade e associação com indicadores de risco ou de proteção para doenças crônicas não transmissíveis. Arq Bras Endocrinol Metabol 2009; 53:1146-1156.

28. Maher CA, Mire E, Harrington DM, Staiano AE, Katzmarzyk PT. The Independent and Combined Associations of Physical Activity and Sedentary Behavior with Obesity in Adults: NHANES 2003-06. Obes Silver Spring Md 2013; 21(12):E730-E737.

29. Silva VS, Souza I, Silva DAS, Fonseca MJM. Prevalence and factors associated with overweight in adults - Brazil, 2008-2009. Rev Bras Cineantropometria Desempenho Hum 2014; 16:161-170.
30. Ward J, Friche AAL, Caiaffa WT, Proietti FA, Xavier CC, Roux AVD. Association of socioeconomic factors with body mass index, obesity, physical activity, and dietary factors in Belo Horizonte, Minas Gerais State, Brazil: The BH Health Study. Cad Saude Publica 2015; 31:182-194.

31. Bilger M, Kruger EJ, Finkelstein EA. Measuring Socioeconomic Inequality in Obesity: Looking Beyond the Obesity Threshold. Health Econ 2017; 26(8):10521066.

32. Santos IKS, Conde WL. Tendência de padrões alimentares entre adultos das capitais brasileiras. Rev Bras Epidemiol 2020; 23:E200035.

33. Baraldi LG, Steele EM, Canella DS, Monteiro CA. Consumption of ultra-processed foods and associated sociodemographic factors in the USA between 2007 and 2012: evidence from a nationally representative cross-sectional study. BMJ Open 2018; 8(3):e020574.

34. Swinburn BA, Sacks G, Hall KD, McPherson K, Finegood DT, Moodie ML, Gortmaker SL. The global obesity pandemic: shaped by global drivers and local environments. Lancet 2011; 378(9793):804-814.

35. Conde WL, Borges C. O risco de incidência e persistência da obesidade entre adultos Brasileiros segundo seu estado nutricional ao final da adolescência. Rev Bras Epidemiol 2011; 14:71-79.

36. Conde WL, Oliveira DR, Borges CA, Baraldi LG. Consistência entre medidas antropométricas em inquéritos nacionais. Rev Saude Publica 2013; 47:69-76.

37. Monteiro CA, Moura EC, Jaime PC, Claro RM. Validade de indicadores do consumo de alimentos e bebidas obtidos por inquérito telefônico. Rev Saude Publica 2008; 42:582-589.

38. Bernal RTI, Malta DC, Claro RM, Monteiro CA. Effect of the inclusion of mobile phone interviews to Vigitel. Rev Saude Publica 2017; 51(Supl. 1):15s.

39. Popkin BM, Adair LS, Ng SW. Global nutrition transition and the pandemic of obesity in developing countries. Nutr Rev 2012; 70(1):3-21.

40. Popkin BM. Nutrition transition and the global diabetes epidemic. Current Diabetes Report 2015; 15:64.

41. Mendonça CP, Anjos LA. Aspectos das práticas alimentares e da atividade física como determinantes do crescimento do sobrepeso/obesidade no Brasil. Cad Saude Publica 2004; 20(3):698-709.

\footnotetext{
Artigo apresentado em 12/08/2019

Aprovado em 18/11/2019

Versão final apresentada em 20/11/2019

Editores-chefes: Romeu Gomes, Antônio Augusto Moura da Silva
} 
